\title{
The effect of different test protocols and walking distances on gait speed in older persons
}

\author{
Sebastian Krumpoch ${ }^{1}$. Ulrich Lindemann ${ }^{2} \cdot$ Anja Rappl $^{3} \cdot$ Clemens Becker $^{2} \cdot$ Cornel C. Sieber $^{1,4} \cdot$ Ellen Freiberger $^{1}$
}

Received: 23 August 2020 / Accepted: 28 August 2020 / Published online: 15 September 2020

(c) The Author(s) 2020

\begin{abstract}
Background and aims Walking is the core physical activity of older persons. The assessment of walking capacity is increasingly important for clinical purposes and clinical research. Differences between assessment tools and protocols for short walks to obtain gait characteristics can be responsible for changes, e.g., in gait speed from 0.1 to $0.2 \mathrm{~m} / \mathrm{s}$. The purpose of this study was to generate further knowledge for the harmonization and/or standardization of short walk-test protocols for assessing gait characteristics under supervised conditions.

Methods For this cross-sectional study, 150 community-dwelling older adults (mean age $80.5 \pm 4.5$ years) were recruited. Participants performed eight walks differing in the distance (8-versus 4-m), static versus dynamic trials and comparing different test speed instructions (usual versus maximal) on an electronic walkway.

Results A meaningful significant difference in mean usual gait speed was documented comparing the 4-m dynamic and static test protocol $(0.12 \mathrm{~m} / \mathrm{s} ; p=0.001)$. For the same comparison over an $8-\mathrm{m}$ distance (dynamic versus static) and for the comparison between usual gait speed over 4-and 8-m, the differences in gait speed were smaller, but still statistically significant $(p=0.001)$.

Conclusions Gait speed was faster, if the test protocol did not include a static start or stop. The differences were greater for a shorter walking distance. This aspect should be considered for the comparison of study results and is particularly relevant for systematic reviews and meta-analyses.
\end{abstract}

Keywords Acceleration phase $\cdot$ Deceleration phase $\cdot$ Gait speed $\cdot$ Aged $\cdot$ Test protocol

\section{Introduction}

Walking is a core activity of older persons and a relevant component to overall mobility. Evidence exists that a decline in gait speed of older persons is associated with many

Sebastian Krumpoch

sebastian.krumpoch@fau.de

1 Institute for Biomedicine of Aging, Friedrich-AlexanderUniversity of Erlangen-Nürnberg, Kobergerstr. 60, 90408 Nuremberg, Germany

2 Department of Geriatrics and Clinic for Geriatric Rehabilitation, Robert-Bosch-Hospital, Auerbachstr. 110, 70376 Stuttgart, Germany

3 Department of Medical Informatics, Biometry and Epidemiology, Friedrich-Alexander-University of Erlangen-Nürnberg, Waldstr. 6, 91054 Erlangen, Germany

4 Department of Medicine, Kantonsspital Winterthur, Brauerstr. 15, 8400 Winterthur, Switzerland negative health outcomes, such as death [1], frailty [2] or hospital admission [3]. Gait speed is increasingly acknowledged as a "vital sign" [4]. To be used in clinical practice, gait speed measurements should be standardized and for clinical research the results should be at least harmonized [5].

Assessing gait speed is not trivial. Gait characteristics can be measured supervised in a research laboratory or unsupervised during daily activities by body-worn sensors [6]. It is increasingly recognized that supervised gait speed in controlled environments differs significantly from real-life nonsupervised gait speed measured in the general older community [7] and according to disease status [8-10]. Monitoring gait speed in real life is not yet established. This requires assessments by body-worn sensors over several days [6]. Gait speed is measured predominantly in the gait laboratory or similar clinical settings. Instruments like electronic walkways or photoelectric barriers are established gold standards. 
They eliminate reaction time errors, which can occur when measuring gait speed manually.

Provided the test methodology is fully reported at all [5], research groups often use walking protocols with different gait conditions to test their study participants $[7,8]$. This includes walking distances of 4-m to 10-m. Most protocols include a normal/usual/habitual walking condition and a fast walking condition without or with additional cognitive tasks. Additionally, standardized measurement of steady-state gait via short tests is problematic, because the real-life walking includes acceleration and deceleration affecting mean gait speed $[11,12]$. For clinical routines, this is impractical as time and resources are limited. Therefore, clinical routines have simplified the gait assessment using only one condition. This plethora of approaches result in a difficult position for clinicians leading to different protocols since the landmark JAMA study by Studenski [1]. Other studies show that differences between assessment tools and protocols can be responsible for changes in supervised gait speed in a range of $0.1-0.2 \mathrm{~m} / \mathrm{s}$ [13-15]. These are speed ranges that are considered as clinically meaningful [16].

The ongoing debate is now being influenced by regulatory agencies such as the European Medicines Agency (EMA) and US Food and Drug Administration. The EMA in a recent decision has adopted the Short Physical Performance Battery (SPPB) [17] as their current gold standard to assess mobility. This includes a 4-m supervised gait assessment from a standing start. It can be expected that this will establish a norm at least for clinical routines and additional testing will use this as a frame to discuss their findings.

The purpose of this study was to generate further knowledge for the harmonization (standardization) of supervised short walk-test protocols as well as data generalization. Additional methodological findings could increase and specify the significance of gait speed measurements, reduce the risk of errors in group- and sample comparisons and facilitate the constitution of standardized protocols in the scientific community [5]. It was hypothesized that usual gait speed is faster, if the test protocol does not include a static start or stop at the end. Furthermore, this study also examined whether this effect could be reproduced with maximal gait speed and if a difference between protocols would be greater for a shorter walking distance.

\section{Methods}

\section{Subjects and design}

For this cross-sectional study, community-dwelling older adults were recruited between May and December 2019 from an existing data pool and by flyers distributed in the greater area of a south-east German city. Participants had to be at least 70 years old, able to walk without a wheeled walker for 10-m and to understand and follow instructions. Exclusion criteria were orthopedic and/or neurologic problems, which caused walking problems (self-report). The study protocol was approved by the ethical committee of FAU Ethical Committee (43_19B) and all participants had to give written informed consent.

\section{Primary outcome measures}

Gait analysis was performed on an instrumented 10-m-long walkway with embedded pressure sensors (Gold walkway, $972 \mathrm{~cm}$ long, active electronic surface area $792 \times 610 \mathrm{~cm}$, total 29,952 pressure sensors, scanning frequency $60 \mathrm{~Hz}$, GAITRite, CIR Systems Inc., Franklin, USA) in a well-lit hallway. The validity and reliability of the GAITRite system has been shown in previous studies [18, 19]. The GAITRite system calculated gait speed as the ratio of the parameters Distance Travelled and Ambulation Time. Distance Travelled was measured on the horizontal axis from the heel center of the first footprint to the heel center of the last footprint, while Ambulation Time was recorded as the time elapsed between first contact of the first and the last footfalls. The protocol consisted of eight walks differing with respect to distance (7.9-versus $4.3-\mathrm{m}$; in the following indicated as 8-and 4-m, respectively), start/end protocols (static versus dynamic) and speed (usual versus maximal) (Table 1). The 8-m-long active sensor area of the instrumented walkway can be modified using the GAITRite software. The 4-m measurements were realized by computer-based reduction of the not required carpet length. Cones were placed on the side of the walkway to visualize the start and finish area for each test condition. The rationale for comparing a 4-m distance with an 8-m distance was the widely used 4-m distance as part of the SPPB [17] and another short, but somewhat longer distance which is also described in the literature [15]. Static walks involved exclusively the active carpet area. Dynamic and semi-dynamic walks included acceleration and deceleration phases of 2-m, which were partly outside the

Table 1 Test conditions of all walks with regard to gait speed, start/ end and distance

\begin{tabular}{llll}
\hline Gait speed & Start condition & End condition & Distance $(\mathrm{m})$ \\
\hline Usual gait speed & Static & Static & 4 \\
Usual gait speed & Dynamic & Dynamic & 4 \\
Usual gait speed & Static & Dynamic & 4 \\
Usual gait speed & Dynamic & Static & 4 \\
Usual gait speed & Static & Static & 8 \\
Usual gait speed & Dynamic & Dynamic & 8 \\
Maximal gait speed & Static & Static & 8 \\
Maximal gait speed & Dynamic & Dynamic & 8 \\
\hline
\end{tabular}


active carpet area (Fig. 1). The instruction for a static walk at usual pace was: "Please walk in your usual gait speed and come to a sudden stop when you have reached the cone." The instruction for a dynamic walk at maximum pace was: "Please walk as quickly and safe as possible without running until you reach the indicated cone." Participants were allowed to rest between walks as needed. The walks were carried out in randomized order.

\section{Descriptive measures/covariates}

Demographic and anthropometric data, such as age, sex, body weight and height were recorded. Functional comorbidity [20] was obtained by questionnaires in a standardized interview. Cognitive function was assessed with the Montreal Cognitive Assessment [21]. The SPPB sum score [17] was used to describe the global physical function of the participants.

\section{Data analysis}

Paired $t$ tests analyzed the differences with regard to the inclusion or exclusion of the acceleration and deceleration on the 8-m walks in usual, as well as in maximal gait speed.

A one-way repeated measure analysis of variance (ANOVA) was used to examine the effect of four different combinations of acceleration and deceleration on gait speed over 4-m.
The effect of the walking distances (4-and 8-m), along with the effects of including or excluding the acceleration and deceleration and their interaction was analyzed by a two-way repeated-measure ANOVA. For all calculations, a significance level at $\alpha=0.05$ was used. Post hoc paired $t$ tests with Bonferroni correction were applied. All analyses were performed using SPSS $^{\circledR}$ version 24.0 (SPSS, Inc., Chicago, IL, USA).

\section{Results}

The data of 150 participants with a mean age of $80.5 \pm 4.5$ years $(61 \%$ women) were included in the analysis. The description of the cohort in detail is presented in Table 2.

A clinically meaningful difference in mean usual gait speed was documented for the comparisons between the 4-m dynamic test protocol and 4-m static test protocol $(0.12 \mathrm{~m} / \mathrm{s})$ and between the 4-m dynamic test protocol and 8-m static test protocol $(0.1 \mathrm{~m} / \mathrm{s})$. All mean gait speeds at different test conditions are presented in Table 3.

Differences between dynamic and static test protocols for both usual $(t(d f=149)=6.96, p=0.001)$ and maximal $(t$ $(d f=149)=4.85, p=0.001)$ gait speed over 8 -m were statistically highly significant.

Comparing the 4-m walks concerning four different test protocols, a repeated measures ANOVA with a Huynh-Feldt
Fig. 1 Description of the test conditions with regard to acceleration and deceleration. 4-m,4-meter; 8-m, 8-meter

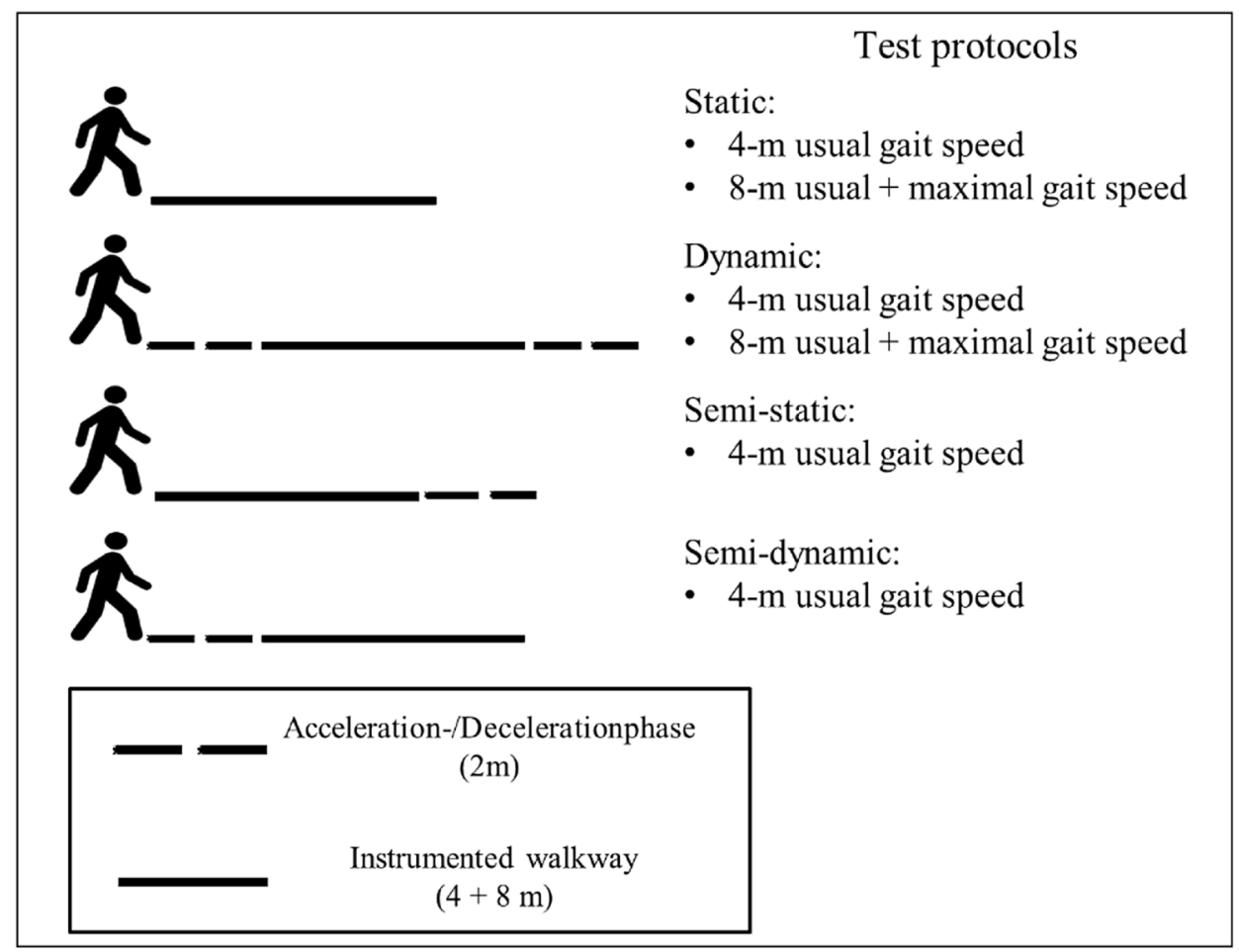

4-m = 4-meter, 8-m = 8-meter 
Table 2 Description of all $(n=150)$ participating older adults

\begin{tabular}{ll}
\hline Characteristic & Mean \pm SD (range) or $n(\%)$ \\
\hline Sex, female/male & $92(61) / 58(39)$ \\
Age [years] & $80.5 \pm 4.5(71-93)$ \\
Body height $[\mathrm{cm}]$ & $163.8 \pm 10.5(142-198)$ \\
Body weight $[\mathrm{kg}]$ & $74.9 \pm 16.2(43.9-121.9)$ \\
Education [years] & $13.7 \pm 3.3(8-28)$ \\
Body mass index $\left[\mathrm{kg} / \mathrm{m}^{2}\right]$ & $27.8 \pm 4.8(17.6-43.3)$ \\
Short Physical Performance Battery & $10.9 \pm 1.7(4-12)$ \\
$\quad(0-\underline{12})$ & \\
Montreal Cognitive Assessment $(0-\underline{30})$ & $25.4 \pm 2.9(13-30)$ \\
Functional Comorbidity Index $(\underline{0}-18)$ & $3.6 \pm 2.2(0-9)$ \\
\hline
\end{tabular}

$S D$ standard deviation; Note: better score values are underlined

correction revealed that mean performance levels showed a statistically significant difference between measurements ( $F$ $(2.83,420.86)=92.67, p<0.001$, partial $\left.\eta^{2}=0.38\right)$. Bonferroni-adjusted post hoc analysis presented significant differences between all 4-m walks (Table 4).

The two-way repeated measures ANOVA considering distance, test protocol (dynamic and static) and the interaction thereof displayed a statistically significant interaction between the effects of walking distance (4-m vs. 8-m) and test protocol (dynamic vs. static). Simple main effect analysis showed that test protocols had a significant impact on gait speed, but there was no overall distance-related difference between 4-m and 8-m walking speed (Table 5). Only when combined, there was a significant effect (interaction $\mathrm{A} \times \mathrm{B}$ ).

\section{Discussion}

As expected, we observed in our study a significant effect of the inclusion or exclusion of acceleration and deceleration phases on gait speed measurements over 4-and 8-m. This effect confirmed our hypothesis and was strongest, i.e., clinically meaningful and statistically significant, between
Table 4 Levels of statistical significance ( $p$ values) of differences between test conditions for 4- $\mathrm{m}$ walks at usual gait speed analyzed by one-way repeated measures analysis of variance

\begin{tabular}{lllll}
\hline & $\mathrm{D} / \mathrm{D}$ & $\mathrm{S} / \mathrm{S}$ & $\mathrm{D} / \mathrm{S}$ & $\mathrm{S} / \mathrm{D}$ \\
\hline $\mathrm{D} / \mathrm{D}$ & & $0.001^{* *}$ & $0.001^{* *}$ & $0.001^{* *}$ \\
$\mathrm{~S} / \mathrm{S}$ & $0.001^{* *}$ & & $0.001^{* *}$ & $0.001^{* *}$ \\
$\mathrm{D} / \mathrm{S}$ & $0.001^{* *}$ & $0.001^{* *}$ & & $0.016^{*}$ \\
$\mathrm{~S} / \mathrm{D}$ & $0.001^{* *}$ & $0.001^{* *}$ & $0.016^{*}$ & \\
\hline
\end{tabular}

$S$ static; $D$ dynamic

*Statistically significant difference in mean walking speed among four conditions with different test protocols; $p<0.05^{*}, p<0.001^{* *}$

Table 5 Two-way repeated measures analysis of variance on: main effects $\mathrm{A}$ and $\mathrm{B}$, interaction effect $\mathrm{A} \times \mathrm{B}$

\begin{tabular}{lrlll}
\hline Effect & \multicolumn{1}{c}{$F$} & $d f$ & Partial $\eta^{2}$ & $p$ \\
\hline Distance (A) & 0.32 & 1 & 0.002 & 0.572 \\
Test protocol (B) & 194.27 & 1 & 0.566 & $0.001^{*}$ \\
A $\times$ B & 17.86 & 1 & 0.107 & $0.001^{*}$ \\
\hline
\end{tabular}

$A$ main effect of 4-m or 8-m walking distance; $B$ main effect of acceleration/deceleration inclusion

$* p<0.001$

4-m walks with uniform test protocols in usual gait speed. Statistically significant differences were attained for 4-m walks with mixed test protocols in usual gait speed and for both $8-\mathrm{m}$ walks with uniform test protocols in maximal and usual gait speed. While the overall distance of walks had no significant effect on usual gait speed, the test protocol as well as the interaction of test protocol and distance did so.

In general, our results are in line with other findings and emphasize the importance of standardized test protocols for supervised gait speed measurements in research and clinical practice. Sustakoski et al., and Wang et al., found clinically meaningful and statistically significant effects of test protocols in similar populations, but with different instrumentation [13, 14]. Warden et al. [15] showed differences for usual
Table 3 Mean gait speeds of different test conditions of all participating older adults $(\mathrm{n}=150)$

\begin{tabular}{llll}
\hline Distance/speed & $\begin{array}{l}\text { Test protocol } \\
\text { Start }\end{array}$ & $\begin{array}{l}\text { Test protocol } \\
\text { End }\end{array}$ & Mean \pm SD (range) [m/s] \\
\hline 4-m usual gait speed & Dynamic & Dynamic & $1.23 \pm 0.28(0.25-2.01)$ \\
4-m usual gait speed & Static & Static & $1.11 \pm 0.26(0.24-1.92)$ \\
4-m usual gait speed & Static & Dynamic & $1.17 \pm 0.26(0.26-1.75)$ \\
4-m usual gait speed & Dynamic & Static & $1.19 \pm 0.28(0.27-1.94)$ \\
8-m usual gait speed & Dynamic & Dynamic & $1.20 \pm 0.26(0.33-1.80)$ \\
8-m usual gait speed & Static & Static & $1.13 \pm 0.27(0.29-1.83)$ \\
8-m maximal gait speed & Dynamic & Dynamic & $1.52 \pm 0.35(0.34-2.39)$ \\
8-m maximal gait speed & Static & Static & $1.47 \pm 0.34(0.31-2.31)$ \\
\hline
\end{tabular}

$S D$ standard deviation; 4-m =4-meter, 8- $\mathrm{m}=8$-meter 
(mean difference $0.05 \mathrm{~m} / \mathrm{s}$ ) and maximum (mean difference $0.16 \mathrm{~m} / \mathrm{s}$ ) gait speed in younger participants. Furthermore, Wang et al. revealed that gait speeds with dynamic protocols were comparable over shorter (4-m) and longer (10-m) distances [14]. In the present study, these findings could not be replicated over 4-and 8-m.

Although all aforementioned studies examined slightly different protocols, they showed a significant effect of the inclusion or exclusion of acceleration and deceleration phases on gait speed measurements. Correspondingly, the comparison of our, seemingly identical, 4-m walk tests with mixed setup conditions (i.e., static-dynamic with dynamicstatic) already yielded significant differences, suggesting a sizeable impact of even marginal test protocol variations. Therefore, the influence of test protocols on gait speed measurements should be considered in future inter-study comparisons and be weighted as potential exclusion criterion in meta-analysis. Alternatively, results must be adjusted. In general, our results indicate that short walk tests, e.g., 4-m, might be more vulnerable to a change in the protocol compared to a longer walk, e.g., 8-m. Due to the regulatory norms propagating the SPPB as a standard measure, it can be expected that many clinicians will adopt the 4-m walk from a standing position as their "gold" standard. This is also likely based on the limited space often available in outpatient settings and general practitioner offices. Consequently, the use of short walk assessments may have a dilutive effect in a meta-analysis.

It is noteworthy that usual gait speed ranged up to $2.01 \mathrm{~m} / \mathrm{s}$ in our cohort of older persons, which could be indicative for the influence of an observer effect. An emerging discussion is the need and possibility to use supervised and non-supervised gait speed in real-world environments as a standard measure. This development is also pushed by the universal deployment of sensors in smartphones and other wearable sensor technology such as wristband sensing. Recent findings show that measurements of supervised usual gait speed in controlled conditions such as outpatient and inpatient clinics are significantly faster than in gait speed measurements in daily life [7]. It is likely that the setting, the observer and contextual circumstances have a major influence on "normal", "usual" or "habitual" walking and gait speed in particular.

\section{Limitations}

To keep the physical burden for participants within tolerable limits and to avoid fatigue effects, the gait analysis was restricted to only eight walks and to only the gait speed parameter. As a result, not every possible test protocol combination over 4-and 8-m was examined. Furthermore, every condition was only assessed once, increasing the variance of the gait speed measurements compared to the mean of multiple trials. Future research could investigate the effect on other kinematic parameters.

The inclusion criteria might have produced a selection effect toward a participation of mainly physical healthy older persons. Additionally, some participants could have distorted the results due to over-motivation. Therefore, our results cannot be considered as a representation of the general population in the age range of $\geq 70$ years, affecting the generalizability of the study.

\section{Conclusion}

Gait speed was faster, if the test protocol did not include a static start or stop and this difference was greater for a shorter walking distance. This aspect must be regarded for comparison of study results and for future meta-analyses.

Acknowledgements This paper was submitted in partial fulfillment of the requirements for obtaining the degree Dr. rer. biol. hum. (PhD in Human Biology) for the primary author.

Author contributions All authors contributed to the study conception and design. Data collection and analysis were performed by Sebastian Krumpoch and Anja Rappl. The first draft of the manuscript was written by Sebastian Krumpoch and Ulrich Lindemann and all authors commented on previous versions of the manuscript. All authors read and approved the final manuscript.

Funding Open Access funding provided by Projekt DEAL. This research did not receive any specific grant from funding agencies in the public, commercial, or not-for-profit sectors.

Availability of data and material The datasets generated during this study are not publicly available, but are available from the corresponding author on reasonable request.

\section{Compliance with ethical standards}

Conflict of interest Author CB is consultant of E. Lilly, Nutritia and Amgen, Robert Bosch Healthcare and Philips Germany. All other authors declare no conflict of interest.

Ethics approval The study was performed in accordance with the ethical standards of the ethical committee of the local university and with the 1964 Helsinki Declaration and its later amendments. This article does not contain any studies with animals performed by any of the authors. This study was approved by the FAU Ethical committee (43_19B).

Informed consent All participants of this project gave written informed consent for data analysis and publication.

Open Access This article is licensed under a Creative Commons Attribution 4.0 International License, which permits use, sharing, adaptation, distribution and reproduction in any medium or format, as long as you give appropriate credit to the original author(s) and the source, 
provide a link to the Creative Commons licence, and indicate if changes were made. The images or other third party material in this article are included in the article's Creative Commons licence, unless indicated otherwise in a credit line to the material. If material is not included in the article's Creative Commons licence and your intended use is not permitted by statutory regulation or exceeds the permitted use, you will need to obtain permission directly from the copyright holder. To view a copy of this licence, visit http://creativecommons.org/licenses/by/4.0/.

\section{References}

1. Studenski S, Perera S, Patel K et al (2011) Gait speed and survival in older adults. JAMA 305:50-58

2. Schoon Y, Bongers K, Van Kempen J et al (2014) Gait speed as a test for monitoring frailty in community-dwelling older people has the highest diagnostic value compared to step length and chair rise time. Eur J Phys Rehabil Med 50:693-701

3. Cesari M, Kritchevsky SB, Penninx BWHJ et al (2005) Prognostic value of usual gait speed in well-functioning older people-results from the Health, Aging and Body Composition Study. J Am Geriatr Soc 53:1675-1680

4. Middleton A, Fritz SL, Lusardi M (2015) Walking speed: the functional vital sign. J Aging Phys Act 23:314-322

5. Graham JE, Ostir GV, Kuo Y-F et al (2008) Relationship between test methodology and mean velocity in timed walk tests: a review. Arch Phys Med Rehabil 89:865-872

6. Zijlstra W, Aminian K (2007) Mobility assessment in older people: new possibilities and challenges. Eur J Ageing 4:3-12

7. Van Ancum JM, van Schooten KS, Jonkman NH et al (2019) Gait speed assessed by a 4-m walk test is not representative of daily-life gait speed in community-dwelling adults. Maturitas 121:28-34

8. Foucher KC, Thorp LE, Orozco D et al (2010) Differences in preferred walking speeds in a gait laboratory compared with the real world after total hip replacement. Arch Phys Med Rehabil 91:1390-1395

9. Storm FA, Nair KPS, Clarke AJ et al (2018) Free-living and laboratory gait characteristics in patients with multiple sclerosis. PLoS ONE 13:e0196463

10. Toosizadeh N, Mohler J, Lei H et al (2015) Motor performance assessment in Parkinson's Disease: association between objective in-clinic, objective in-home, and subjective/semi-objective measures. PLoS One 10:e124763
11. Lindemann U, Najafi B, Zijlstra W et al (2008) Distance to achieve steady state walking speed in frail elderly persons. Gait Posture 27:91-96

12. Macfarlane PA, Looney MA (2008) Walkway length determination for steady state walking in young and older adults. Res Q Exerc Sport 79:261-267

13. Sustakoski A, Perera S, VanSwearingen JM et al (2015) The impact of testing protocol on recorded gait speed. Gait Posture 41:329-331

14. Wang C-Y, Chen T-R, Lin Y-H et al (2012) Gait speed measure: the effect of different measuring distances and the inclusion and exclusion of acceleration and deceleration. Percept Mot Skills 114:469-478

15. Warden SJ, Kemp AC, Liu Z et al (2019) Tester and testing procedure influence clinically determined gait speed. Gait Posture 74:83-86

16. Bohannon RW, Glenney SS (2014) Minimal clinically important difference for change in comfortable gait speed of adults with pathology: a systematic review. J Eval Clin Pract 20:295-300

17. Guralnik JM, Simonsick EM, Ferrucci L et al (1994) A short physical performance battery assessing lower extremity function: association with self-reported disability and prediction of mortality and nursing home admission. J Gerontol 49:M85-M94

18. Bilney B, Morris M, Webster K (2003) Concurrent related validity of the GAITRite walkway system for quantification of the spatial and temporal parameters of gait. Gait Posture 17:68-74

19. Menz HB, Latt MD, Tiedemann A et al (2004) Reliability of the GAITRite walkway system for the quantification of temporospatial parameters of gait in young and older people. Gait Posture 20:20-25

20. Groll DL, To T, Bombardier C et al (2005) The development of a comorbidity index with physical function as the outcome. J Clin Epidemiol 58:595-602

21. Nasreddine ZS, Phillips NA, Bédirian V et al (2005) The Montreal Cognitive Assessment, MoCA: a brief screening tool for mild cognitive impairment. J Am Geriatr Soc 53:695-699

Publisher's Note Springer Nature remains neutral with regard to jurisdictional claims in published maps and institutional affiliations. 